\title{
Creencias y rendimiento académico en el aprendizaje de las lenguas extranjeras
}

\author{
María Dolores Corpas Arellano \\ Consejería de Educación de la Junta de Andalucía \\ Granada, España
}

\begin{abstract}
Resumen
Todos tenemos creencias que determinan nuestras predisposiciones, decisiones, comportamientos y acciones en la vida. Esta investigación empírica pretende analizar las creencias del alumnado español de cuarto nivel de Educación Secundaria Obligatoria con respecto al proceso de enseñanza-aprendizaje de la lengua extranjera. El objetivo principal es establecer una correlación entre las creencias y el rendimiento académico del alumnado de inglés como lengua extranjera. El estudio concluye afirmando que aquellos estudiantes que logran los mejores resultados creen que el inglés es fácil de aprender, es útil para sus estudios posteriores y les gusta aprenderlo. Esto está relacionado con una alta motivación intrínseca del alumnado, lo que favorece el aprendizaje autodirigido y les permite buscar nuevos desafíos en este proceso.
\end{abstract}

Palabras claves: creencias, rendimiento, aprendizaje, lengua extranjera

\begin{abstract}
We all have beliefs which determine our willingness, decisions, behaviours, and actions in our lives. This empirical research analyzes Spanish students' beliefs in their $4^{\text {th }}$ year of Compulsory Secondary Education (CSE) in relation to the foreign language teaching-learning process. The main aim is to establish a correlation between students' beliefs and their achievement in English as a foreign language. This study concludes affirming that those students who achieve the best outcome believe that English is easy to learn, it is useful for their higher education, and they love learning English. These beliefs are related to a high intrinsic motivation, which contributes to reinforce students' self-directed learning and allows them to find new learning challenges.
\end{abstract}

Key words: beliefs, achievement, learning, foreign language 


\section{Introducción}

Dodos tenemos creencias y éstas son determinantes para nuestra vida, tanto que influyen en nuestras decisiones categóricamente. De la misma manera, nuestras creencias reciben influencia constante de factores contextuales que nos incitan a actuar en una determinada dirección. Un ejemplo son las creencias políticas, religiosas y las creencias que nosotros tenemos sobre nuestro entorno y nosotros mismos. Ortega y Gasset (1959) subraya que las creencias no son ideas que tenemos, sino ideas que somos. Para el individuo, las creencias se confunden con la realidad y, consecuentemente, se considera que esas creencias forman parte de la misma realidad, aunque sean creencias erróneas.

La naturaleza y las funciones de las creencias y sus relaciones con los conceptos de las actitudes, intenciones $\mathrm{y}$ acciones han sido ampliamente estudiadas por distintas disciplinas como la filosofía, la educación, la sociología, la psicología, la pedagogía y la publicidad. En el aprendizaje de lenguas extranjeras, los estudios sobre las creencias del profesorado y del alumnado se remontan a los años setentas. Desde entonces, se han publicado numerosas investigaciones y parece existir un consenso entre la comunidad científica y el ámbito educativo en cuanto a la relevancia de las creencias de los sujetos participantes en el proceso de enseñanza-aprendizaje de una lengua extranjera. Tanto el alumnado como el profesorado tienen creencias previas sobre cómo se aprende una lengua extranjera y qué factores influyen en su aprendizaje.

Así, las creencias individuales que el alumnado tiene sobre el aprendizaje de una lengua pueden influir, consciente o inconscientemente, en su aproximación a esta lengua. Horwitz (1999) subraya la importancia de comprender las creencias de los estudiantes para entender mejor la forma de abordar el aprendizaje de una lengua y el empleo de estrategias de aprendizaje para planificar adecuadamente la instrucción. Precisamente, un uso adecuado de las estrategias de aprendizaje es esencial para el desarrollo óptimo de las destrezas lingüísticas y comunicativas (Corpas, 2008 y 2010).

\section{Creencias en el aprendizaje de una lengua}

\subsection{El concepto de creencia}

Las creencias en el aprendizaje de una lengua se refieren a las nociones, ideas percibidas, conceptos, opiniones, representaciones, suposiciones o miniteorías acerca de la naturaleza de la lengua o del aprendizaje de la lengua (Holec, 1981 y Horwitz, 1987). Se piensa, generalmente, que los estudiantes de una lengua sostienen diferentes creencias sobre cómo se aprende, qué factores influyen de manera positiva en su aprendizaje y para qué sirve aprender una lengua.

Woods (1996) define el concepto de creencia como la aceptación de una proposición para la cual no existe conocimiento convencional, que no es demostrable y para la cual existe desacuerdo conocido. Para Richardson (1996), las creencias son formas de comprender premisas o proposiciones sobre el mundo, sostenidas psicológicamente, que son tenidas por verdaderas.

Las creencias individuales sobre el aprendizaje de una lengua pueden 
conscientemente o inconscientemente influenciar las aproximaciones o comportamientos en su aprendizaje. Por ejemplo, los estudiantes que valoran la importancia de aprender gramática se centrarán en las estructuras de una oración o en las fórmulas del lenguaje informal. Horwitz (1999) insiste en que es sumamente importante comprender las creencias de los estudiantes para entender mejor el acercamiento al aprendizaje de una lengua y al empleo de estrategias de aprendizaje para planificar adecuadamente la instrucción en esa lengua.

Williams y Burden (1999) también consideran de suma importancia, las creencias de los estudiantes en el aprendizaje de una lengua, y afirman que las creencias inciden más que los conocimientos a la hora de determinar la forma en que los individuos organizan y defienden tareas y problemas, y predicen con más precisión la forma en que los profesores se comportan en el aula.

Ramos (2010) recoge algunas creencias ampliamente extendidas sobre el aprendizaje de una lengua extranjera, las cuales han pasado a formar parte de la sabiduría popular y de nuestro acervo común de conocimientos sobre lo que es o debe ser aprender una lengua extranjera:

- Los niños aprenden lenguas con más facilidad que los adultos.

- Como mejor se aprende una lengua es en el país donde ésta se habla.

- Las personas que hablan más de una lengua son muy inteligentes.

- La gente que es buena en matemáticas o ciencias no lo es para aprender una lengua.

- Para aprender una lengua hay que practicar mucho.

\subsection{Características de las creencias}

A continuación se exponen las características de las creencias según Williams y Burden (1997):

- Son resistentes al cambio. Las creencias son muy estables aunque pueden ser influenciables bajo entrenamiento específico.

- Son difíciles de medir. Se utiliza la deducción a partir del comportamiento de las personas mediante un análisis de lo que los aprendices dicen y hacen. Obviamente, las creencias no se pueden medir por procedimientos psicométricos ni observar directamente. En ocasiones, las creencias de los estudiantes aparecen de forma explícita; no obstante, en la mayoría de las ocasiones hay que deducirlas de los datos recogidos.

- Tienden a estar limitadas por la cultura en la que el individuo se desarrolla. Indudablemente, la cultura en la que una persona está inmersa influye en sus creencias aunque no las determina de manera concluyente. Otros factores como la cultura del aula también inciden en las creencias.

- Las creencias están interrelacionadas con los conocimientos que creemos que tenemos, a pesar de que actúan como un filtro sobre el pensamiento y el procesamiento de la información. Así, las creencias de un alumno inciden en la manera de percibir la enseñanza que recibe y su propio proceso de aprendizaje.

- Las creencias están interrelacionadas entre sí y con otros aspectos como las actitudes y valores de una persona. Las creencias se presentan organizadas en sistemas, no de forma aislada. 
Espinosa (2009) añade los siguientes rasgos:

- Las características de las creencias son dinámicas puesto que cambian en el tiempo y en una misma situación.

- Emergentes, socialmente construidas y contextualizadas. No permanecen como estructuras mentales listas y fijas, sino que cambian y se desarrollan a medida que interactuamos y modificamos nuestras experiencias y somos, al mismo tiempo, modificados por ellas. Nacen en el contexto de la interacción y en la relación con los grupos sociales.

- Frutos de la experiencia. Todos los procesos cognitivos, incluido el lenguaje, que nacen de la naturaleza contextual de la existencia humana y de la experiencia entendida esta última como resultado de las interacciones entre el individuo y el ambiente, entre aprendices y entre aprendices y profesores.

- Mediadoras, ya que pueden ser consideradas como instrumentos y herramientas disponibles que se pueden usar o no dependiendo de la situación, tarea y personas que interactúan.

- Paradójicas y contradictorias. Pueden actuar como instrumentos para facilitar o dificultar el proceso de enseñanza-aprendizaje. Son sociales pero también individuales y únicas. Son compartidas, emocionales, diversas y uniformes.

- Relativas a la acción de una manera indirecta y compleja.

- Difíciles de distinguir del conocimiento. No se separan fácilmente de otros aspectos como conocimiento, motivación y estrategias de aprendizaje.

Los autores Ajzen y Fishbein (2000) añaden la cualidad "fuerza" para distinguir unas creencias de otras dentro del sistema de creencias de cada individuo. Así, las creencias más fuertes son consecuencia de la experiencia directa y están relacionadas con la identidad. Además, la fuerza de una creencia es directamente proporcional a la probabilidad subjetiva de que un objeto tenga un determinado atributo.

\section{Investigaciones sobre las creen- cias en el aprendizaje de una len- gua extranjera}

Las investigaciones sobre las creencias en el aprendizaje de una lengua extranjera pueden dividirse en tres grupos: investigaciones sobre las creencias del profesorado, del alumnado y de ambos sobre el aprendizaje de una lengua extranjera.

\section{a. Investigaciones sobre las creen- cias del profesorado sobre el apren- dizaje de una lengua extranjera}

Según Espinosa (2009), las investigaciones sobre las creencias del profesorado con respecto al aprendizaje de una lengua se han centrado en la identificación y caracterización (Barcelos, 1995), en la relación entre las creencias y la práctica docente (Basturkmen, Loewen y Ellis, 2004) y su cambio y evolución (Peacock, 2001). El mismo Espinosa añade que los resultados de estas investigaciones no son conclusivos en cuanto a las creencias declaradas y la práctica docente, sino que mantienen una naturaleza conflictiva e interactiva. Por ejemplo, el quehacer del docente no siempre refleja sus teorías implícitas. El contexto, las expectativas de los alumnos sobre la clase y las de los profesores sobre el alumnado, la 
programación didáctica, el material, la competencia limitada de los alumnos y la disponibilidad de recursos, entre otros factores influyen en la actuación del profesorado (Barcelos, 2006).

\section{b. Investigaciones sobre las creen- cias del alumnado sobre el aprendi- zaje de una lengua extranjera}

Kunt (1999) estudió las creencias sobre el aprendizaje de la lengua y la ansiedad. El test BALLI fue distribuido a estudiantes universitarios hablantes de turco que estudiaban inglés como lengua extranjera en programas preuniversitarios de inglés en Chipre. El estudio identificó tres categorías de creencias de los estudiantes de turco basadas en el factor de análisis: el valor y la naturaleza del aprendizaje del inglés, la confianza en sí mismo en el habla, y las creencias sobre la interacción social. Los resultados del estudio confirman una alta motivación instrumental para los aprendices del inglés y para los grupos, fuertes creencias en la importancia del inglés y un alto valor localizado en la suposición y la repetición durante la práctica. Además, el estudio reveló correlaciones significativas entre la confianza en sí mismo en el habla y la ansiedad en la lengua extranjera, indicando que cuanto más confianza tienen los estudiantes, menor es su ansiedad.

En el estudio de Diab (2000), una versión modificada de BALLI $^{1}$ fue pasada a estudiantes libaneses que aprendían inglés y francés como lenguas extranjeras en tres universidades del Líbano, para describir las creencias del aprendiz sobre el aprendizaje de la lengua. Los factores identificaron cuatro categorías de creencias para cada grupo de lengua. Las creencias de los estudiantes de inglés eran: motivación integradora, dificultad para hablar y aprender inglés, importancia de la precisión hablando inglés e importancia del inglés en el Líbano. Las creencias de los estudiantes de francés eran: motivación y confianza al hablar francés, la naturaleza del aprendizaje del francés en el Líbano y la importancia y la precisión al hablar francés. Los participantes confirmaron una variedad de creencias sobre las lenguas extranjeras al afirmar que el aprendizaje de una lengua extranjera parece estar relacionado con el contexto político y sociocultural de la educación de la lengua extranjera en el Líbano. Como conclusión, el estudio reveló varias similitudes y diferencias entre las creencias de los grupos. Por ejemplo, la mayoría de los participantes suscribía, claramente, el concepto de la jerarquía de la dificultad de aprender una lengua y valoraba el inglés como una lengua fácil o muy fácil, pero valoraba el francés como una lengua difícil. Sin embargo, más estudiantes de francés que de inglés creían que es mejor aprender una lengua extranjera en un país donde se hable la lengua. El investigador notó que las diferencias observadas en comparación con las creencias de los estudiantes sobre el aprendizaje del inglés y sobre el aprendizaje del francés podían ser el resultado de una variación en las creencias de un grupo particular sobre el aprendizaje de una lengua objeto diferente.

Corpas (2011) investigó la relación entre el rendimiento en la materia de inglés como lengua extranjera y las creencias que el alumnado tenía sobre su propio futuro académico al término de la Educación Secundaria Obligatoria, cuando el alumnado tiene dieciséis 
años y debe decidir si continuar con sus estudios o, en su caso, qué estudios cursar. La mayoría del alumnado se decanta por continuar sus estudios hasta terminar una carrera universitaria media $(26,6 \%)$, o hasta concluir sus estudios de ciclo formativo de grado medio $(25,5 \%)$. La investigación afirma que existe una correlación positiva entre las expectativas de estudios del alumnado y la consecución de los objetivos propuestos en el curso para la asignatura de lengua extranjera. Así, cuanto mayor es la expectativa de estudios del alumnado, más alto es su rendimiento académico. Aquel alumnado con una expectativa alta hacia sus estudios y, por lo tanto, con mejores resultados académicos, desarrolla una motivación intrínseca elevada. Ésta se caracteriza por proceder del propio individuo, estar bajo su control y por ser capaz para autorreforzarse.

Espinosa (2009) resume las conclusiones de otros estudios sobre las creencias del alumnado. Así, los estudiantes tienen creencias sobre una amplia gama de aspectos relativos a la naturaleza de la lengua y su aprendizaje y, además, reciben una fuerte influencia de las experiencias de aprendizaje. Por último, existe una estrecha relación entre el sistema de creencias de aprendizaje de los alumnos y sus estrategias de aprendizaje y las estrategias y acciones que desarrollan en el proceso de aprender una lengua extranjera.

\section{c. Investigaciones sobre las creencias del profesorado y alumnado acerca del aprendizaje de una lengua extranjera}

En el campo de la enseñanza-aprendizaje de lenguas, las investigaciones que hacen partícipes al alumnado $\mathrm{y}$ al profesorado han estudiado, principalmente, aspectos generales como la evaluación, la gramática y corrección de errores y la motivación. Las investigaciones sobre las creencias del profesorado y del alumnado sobre el aprendizaje de una la lengua se centran en:

- La relación entre profesores y alumnos (Barcelos, 2000; Kern, 1995).

- Los conflictos sobre la utilidad de las actividades desarrolladas en clase (Peacock, 1999).

Estos estudios concluyen que las creencias del profesorado y del alumnado son antagónicas y discrepantes en cuanto a sus respectivos papeles, la metodología y la influencia recíproca entre las creencias de ambos.

\section{Características de la investigación}

\section{1. Tipo de investigación}

La investigación que presentamos es de tipo descriptivo pues su objetivo principal es describir sistemáticamente hechos y características de una población o área de interés de forma objetiva y comprobable.

Nuestra investigación es de tipo encuesta porque, conforme con Cohen y Manion (1990: 124), se ocupa de describir las condiciones existentes, identificar valores estándares con los que se pueden comparar las condiciones y determinar las relaciones entre eventos específicos.

\section{2. Objetivos científicos}

En esta investigación, pretendemos dar respuesta a los siguientes objetivos: - Conocer las creencias del alumnado con respecto a la lengua inglesa. 
- Establecer correlaciones entre las creencias del alumnado y su desarrollo académico según los siguientes objetivos curriculares:

- Adquirir la capacidad de comunicarse.

- Comprender textos orales y escritos.

- Producir mensajes orales y escritos.

- Leer de forma comprensiva y autónoma.

- Transferir las estrategias de comunicación de L1 (lengua materna) a L2 (lengua extranjera).

- Reflexionar sobre el funcionamiento de la lengua extranjera.

- Usar estrategias de aprendizaje y recursos didácticos.

- Reflexionar sobre los procesos de aprendizaje.

- Apreciar y reaccionar ante los usos imaginativos y creativos en L2.

- Apreciar los significados sociales y culturales en L2.

- Valorar la importancia de la lengua inglesa.

\section{3. Muestra}

La muestra es el conjunto de individuos representativos de la población objeto de estudio conforme con un procedimiento específico y reunidos como representación válida.

En esta investigación, la muestra invitada está compuesta por los 100 alumnos y alumnas que cursaban la materia de inglés como lengua extranjera en cuarto de ESO, cuando el alumnado tenía entre quince y dieciséis años y estaba a punto de finalizar su educación obligatoria.

La muestra mermó en una alumna porque abandonó los estudios semanas antes de que la prueba fuera realizada. Otros cinco alumnos no completaron todas y cada una de las pruebas por no asistir a clase los días en los que estas se realizaron. Por esta razón, 94 alumnos (48 alumnas y 46 alumnos) forman la muestra real.

En este estudio, ha participado alumnado de tres centros educativos del sur de España. El contexto socioeconómico de estos Institutos de Educación Secundaria (IES) es de clase media y de pequeñas y medias poblaciones (hasta 35000 habitantes). Los padres, madres y tutores de este alumnado son, principalmente, obreros, trabajadores por cuenta propia y trabajadores liberales. Su nivel educativo promedio es de una educación primaria finalizada o estudios profesionales medios.

En cuanto al agrupamiento del alumnado en el aula de lengua extranjera inglés, se siguen las pautas de la Ley Orgánica de Educación (LOE) de 2006 , en la que se afirma que los grupos deben estar compuestos por entre veinticinco y treinta alumnos y alumnas. Éstos deben recibir cuatro horas de inglés semanales impartidas por un especialista en la materia. La mayoría del alumnado de esta investigación empezó a estudiar inglés a la edad de ocho años, tal y como exigía la anterior Ley Orgánica General del Sistema Educativo (LOGSE) de 1990.

\section{4. Instrumentos para la recolec- ción de datos}

En esta investigación hemos utilizado cuestionarios y tests. Los primeros ofrecen datos referidos a la metodología empleada en clase de lengua inglesa y a las características del alumnado y su entorno. La mayoría de las preguntas que se presentan son de elección múltiple para que el alumnado pueda indicar la opción que se ajuste a sus circunstancias. En otras, tiene que 
expresar su opinión usando una escala graduada del uno al cinco, donde el uno es el valor más negativo y cinco el más positivo.

Para conocer el grado de logro en la consecución de distintas tareas y, de esta manera, obtener una nota global partiendo de unas pruebas homogéneas, se han usado tests de lengua.

Las pruebas han sido diseñadas partiendo de los libros de texto vigentes para este nivel educativo y según sus objetivos y contenidos.

\subsubsection{Descripción de las pruebas}

\section{4. 1. 1. Tests de comprensión es- crita (Reading)}

La prueba de comprensión escrita ha sido diseñada teniendo en cuenta los siguientes puntos:

Objetivo que evalúa: Los alumnos deben leer textos adaptados de diferentes tipos e intencionalidad, mostrar comprensión global y específica de los textos y apreciar su estética y figuras literarias. También deben inferir el significado según el contexto.

Formato: La prueba consiste en la realización de cinco tareas. Cada una de ellas contiene un texto y diferentes actividades de comprensión.

Extensión: El número de palabras que contiene la prueba es de alrededor de 600. Cada texto contiene entre 57 y 145 palabras.

Número de items: diecinueve.

Duración: cuarenta y cinco minutos.

Evaluación de la prueba: A fin de evaluar la comprensión escrita, hemos empleado una escala graduada, numerada y creciente de uno a cinco. El valor más negativo es el uno y el más positivo el cinco.

\section{4. 1. 2. Expresión escrita (Writing)}

\section{a. Prueba de expresión escrita}

La prueba de expresión escrita ha sido diseñada teniendo en cuenta los siguientes puntos:

Objetivo que evalúa: El alumnado debe producir, de forma eficaz, distintos tipos de textos con diversas finalidades, en situaciones pasadas, habituales o futuras de comunicación utilizando tanto recursos lingüísticos como no lingüísticos.

Formato: La prueba consta de diez tareas.

Extensión de los textos: El número de palabras que el alumno debe producir nunca excede las 100 palabras en cada tarea.

Número de preguntas: En total, son 31.

Duración: Cincuenta y cinco minutos.

Evaluación de la prueba: Los parámetros evaluados en cada tarea se califican en una escala graduada creciente de uno a cinco, en la cual uno es el valor más negativo y cinco el más positivo

\section{b. Características de tarea de ex- presión escrita Project}

Objetivo que evalúa: Transferir a la lengua extranjera las estrategias de comunicación adquiridas en la lengua materna y utilizar estrategias de aprendizaje y recursos didácticos para narrar acontecimientos.

Formato: Presentada una pregunta el alumno debe resolverla.

Tipo de texto: Texto descriptivo y narrativo.

Actividad: El alumno debe describir o narrar acontecimientos relevantes para el mundo o para su país ocurridos en la década en la que nació. Para documentarse, puede utilizar recursos didácticos 
como enciclopedias, libros de historia, Internet y otros materiales de referencia.

Número de preguntas: Solamente una.

4. 4. 1. 3. Test de comprensión oral (Listening)

La prueba de comprensión oral ha sido diseñada teniendo en cuenta los siguientes puntos:

Objetivo que evalúa: El alumnado debe escuchar textos adaptados con diferente intencionalidad y estructura con el objetivo de mostrar comprensión tanto de los aspectos generales como específicos y apreciar su estética, además de inferir el significado gracias al contexto.

Formato: La prueba consta de seis tareas. Cada una de ellas contiene un texto oral y preguntas relativas a lo escuchado. Una misma tarea puede incluir más de un texto corto relacionados entre sí conforme con la temática.

Extensión de los textos: El número de palabras de la prueba es de 643 . Los textos varían de extensión según el tipo de tarea propuesta, de 26, el más corto, a 179 el más largo.

Número de preguntas: El número total de ítems es de 12.

Duración: Cuarenta minutos.

Evaluación de la prueba: Hemos utilizado una escala graduada creciente de uno a cinco, donde uno es el valor más negativo y cinco el más positivo en cada uno de los parámetros de cada tarea.

\section{4. 1. 4. Test de expresión oral (Speaking)}

La prueba de expresión oral ha sido diseñada teniendo en cuenta los siguientes puntos:

Objetivo que evalúa: Los alumnos deberán responder una serie de pre- guntas que expresen situaciones habituales de comunicación utilizando distintos recursos.

Formato: La prueba consiste en nueve tareas. La extensión de cada una de ellas es distinta. La más corta tiene una sola pregunta, mientras que las más largas incluyen cuatro.

Extensión de los textos: La extensión de los textos varía según el tipo y cantidad de preguntas. Cada texto puede contener entre 40 y 100 palabras aproximadamente.

Número de preguntas: El número de preguntas es de veinte en total.

Duración: La duración de la prueba oscila entre los quince y los veinte minutos aproximadamente.

Evaluación de la prueba: Para la evaluación de esta prueba hemos utilizado una escala graduada, creciente y numerada del uno al cinco. El uno representa el valor más negativo y el cinco el valor más alto

\section{5. Validez y fiabilidad}

La validez de la pruebas se apoya en el juicio de expertos y por la prueba piloto. A fin de determinar la fiabilidad de nuestras pruebas, hemos aplicado el Modelo Alpha de Cronbach ${ }^{2}$, en aquellas pruebas donde ha sido posible, por haber utilizado una escala para evaluar.

\section{6. Estudio estadístico}

Para el estudio estadístico, hemos utilizado la versión 14.0 del programa SPSS (Statistical Package for the Social Science) para Windows. 


\section{Resultados}

\section{1. Creencias y valoración del alum- nado sobre la lengua extranjera}

a) Me gusta aprender inglés

\begin{tabular}{llrrrr}
\multicolumn{6}{c}{ Me gusta aprender inglés } \\
\hline \multicolumn{7}{c}{ Frecuencia } & Porcentaje & $\begin{array}{c}\text { Porcentaje } \\
\text { Válido }\end{array}$ & $\begin{array}{r}\text { Porcentaje } \\
\text { acumulado }\end{array}$ \\
\hline Válidos & Nada & 5 & 5,3 & 5,3 & 5,3 \\
& Poco & 9 & 9,6 & 9,6 & 14,9 \\
& Algo & 40 & 42,6 & 42,6 & 57,4 \\
& Bastante & 29 & 30,9 & 30,9 & 88,3 \\
& Mucho & 11 & 11,7 & 11,7 & 100,0 \\
& Total & 94 & 100,0 & 100,0 & \\
\hline
\end{tabular}

Una amplia mayoría del alumnado, concretamente el 85,2\%, confirma que le gusta aprender inglés, aunque con distinto grado. Un 42,6\% declara que le gusta algo aprender inglés. El $30,9 \%$ dice que le gusta bastante y un $11,7 \%$ afirma que le gusta mucho. Sólo un $5,3 \%$ de la muestra confiesa que no le gusta aprender inglés nada y a un $9,6 \%$ le gusta poco.

\section{b) Aprender inglés es fácil}

\begin{tabular}{llrrrr}
\multicolumn{6}{c}{ Aprender inglés es fácil } \\
\hline \multicolumn{7}{c}{ Frecuencia } & Porcentaje & $\begin{array}{c}\text { Porcentaje } \\
\text { válido }\end{array}$ & $\begin{array}{l}\text { Porcentaje } \\
\text { acumulado }\end{array}$ \\
\hline Válidos & Nada & 14 & 14,9 & 14,9 & 14,9 \\
& Poco & 28 & 29,8 & 29,8 & 44,7 \\
& Algo & 37 & 39,4 & 39,4 & 84,0 \\
& Bastante & 10 & 10,6 & 10,6 & 94,7 \\
& Mucho & 5 & 5,3 & 5,3 & 100,0 \\
& Total & 94 & 100,0 & 100,0 & \\
\hline
\end{tabular}

Un alto porcentaje de los participantes en la investigación cree que aprender inglés no es fácil (14,9\%) o poco fácil (29,8\%). Un 39,4\% piensa que aprender inglés es medianamente fácil. Sin embargo, el 10,6\% del alumnado afirma que es bastante fácil aprender inglés. Tan sólo el 5,3\% del total del alumnado opina que aprender inglés es muy fácil.

\section{c) Me gustan las actividades de la clase de inglés}

\begin{tabular}{lrrrrr}
\multicolumn{5}{c}{ Me gustan las actividades de la clase de inglés } \\
\hline \multicolumn{7}{c}{ Frecuencia } & Porcentaje & $\begin{array}{c}\text { Porcentaje } \\
\text { válido }\end{array}$ & $\begin{array}{c}\text { Porcentaje } \\
\text { acumulado }\end{array}$ \\
\hline Válidos & Nada & 17 & 18,1 & 18,1 & 18,1 \\
& Poco & 30 & 31,9 & 31,9 & 50,0 \\
& Algo & 34 & 36,2 & 36,2 & 86,2 \\
& Bastante & 12 & 12,8 & 12,8 & 98,9 \\
& Mucho & 1 & 1,1 & 1,1 & 100,0 \\
& Total & 94 & 100,0 & 100,0 & \\
\hline
\end{tabular}

Un $18,1 \%$ del alumnado confiesa que no le gustan nada las actividades de la clase de inglés. Un 31,9\% responde que estas actividades le gustan poco. La opción más elegida, con un $36,2 \%$, es la que sostiene que al alumnado le gustan algo las actividades. Sólo un $12,8 \%$ declara que le gustan bastante y un $1,1 \%$ mucho.

\section{d) El inglés es útil para conocer otras formas de vida, etc.}

\begin{tabular}{lrrrrr}
\multicolumn{5}{c}{ El inglés es útil para conocer otras formas de vida, etc. } \\
\hline & & Frecuencia & Porcentaje & $\begin{array}{c}\text { Porcentaje } \\
\text { válido }\end{array}$ & $\begin{array}{r}\text { Porcentaje } \\
\text { acumulado }\end{array}$ \\
\hline Válidos & Nada & 1 & 1,1 & 1,1 & 1,1 \\
& Poco & 9 & 9,6 & 9,6 & 10,6 \\
& Algo & 21 & 22,3 & 22,3 & 33,0 \\
& Bastante & 31 & 33,0 & 33,0 & 66,0 \\
& Mucho & 32 & 34,0 & 34,0 & 100,0 \\
Total & 94 & 100,0 & 100,0 & \\
\hline
\end{tabular}

Los participantes están de acuerdo, de manera terminante, con la utilidad del inglés para conocer otras formas de vida, etc. Así el 33\% y el 34\% valoran este hecho como bastante útil y muy útil, respectivamente. Un $22,3 \%$ de la muestra se sitúa en una posición intermedia. Solo un 9,6\% afirma que es poco útil, mientras que un $1,1 \%$ considera que no es útil. 
e) El inglés es útil en viajes y estancias en otros países

El inglés es útil en viajes y estancias en otros países

\begin{tabular}{llrrrr}
\hline & & Frecuencia & Porcentaje & $\begin{array}{c}\text { Porcentaje } \\
\text { válido }\end{array}$ & $\begin{array}{r}\text { Porcentaje } \\
\text { acumulado }\end{array}$ \\
\hline Válidos & Poco & 3 & 3,2 & 3,2 & 3,2 \\
& Algo & 6 & 6,4 & 6,4 & 9,6 \\
& Bastante & 24 & 25,5 & 25,5 & 35,1 \\
& Mucho & 61 & 64,9 & 64,9 & 100,0 \\
& Total & 94 & 100,0 & 100,0 & \\
\hline
\end{tabular}

Parece haber un amplio consenso entre el alumnado entrevistado en cuanto a la utilidad del inglés en el extranjero. Así, más de dos tercios del alumnado, un 64,9\%, valora que el inglés es muy útil en viajes y estancias en otros países. Un 25,5\%, opina que es bastante útil. El resto del alumnado piensa que es algo útil, un 6,4\%, о росо útil, un 3,2\%. Es importante reseñar que ningún alumno se ha inclinado por la opción más negativa, nada útil.

\section{f) El inglés es útil para mis estudios posteriores}

Casi la mitad del alumnado, un $45,7 \%$, responde que el inglés es muy útil para sus estudios posteriores. A este porcentaje, le sigue el grupo de alumnos que lo considera bastante útil, un $31,9 \%$. Los porcentajes de las demás opciones son, en relación, muy bajos. Un 11,7\% lo valora con una utilidad media. Un 7,4\% piensa que es poco útil para sus estudios posteriores y un $3,2 \%$ piensa que no es útil.

\begin{tabular}{|c|c|c|c|c|c|}
\hline \multicolumn{6}{|c|}{ El inglés es útil para mis estudios posteriores } \\
\hline & & Frecuencia & Porcentaje & $\begin{array}{c}\text { Porcentaje } \\
\text { válido }\end{array}$ & $\begin{array}{l}\text { Porcentaje } \\
\text { acumulado }\end{array}$ \\
\hline \multirow[t]{6}{*}{ Válidos } & Nada & 3 & 3,2 & 3,2 & 3,2 \\
\hline & Poco & 7 & 7,4 & 7,4 & 10,6 \\
\hline & Algo & 11 & 11,7 & 11,7 & 22,3 \\
\hline & Bastante & 30 & 31,9 & 31,9 & 54,3 \\
\hline & Mucho & 43 & 45,7 & 45,7 & 100,0 \\
\hline & Total & 94 & 100,0 & 100,0 & \\
\hline
\end{tabular}

Existe un gran porcentaje de estudiantes que valoran, enormemente, la utilidad de la lengua inglesa para distintas actividades como interacción con otras culturas, viajes y estancias en otros países y para sus estudios posteriores, como acabamos de ver en estas tres últimas preguntas.

\section{2. Correlaciones entre el rendi- miento y las creencias del alumnado sobre la lengua extranjera}

\begin{tabular}{|c|c|c|c|c|c|c|c|c|c|c|c|}
\hline Creencias y valoraciones: & 1 & 2 & 3 & 4 & 5 & 6 & 7 & 8 & 9 & 10 & 11 \\
\hline Me gusta aprender inglés & & & + & & & + & & + & + & + & \\
\hline Aprender inglés es fácil & + & + & + & + & + & & + & & + & & + \\
\hline Me gustan las actividades de la clase de inglés & & & & & & & & & + & + & \\
\hline $\begin{array}{l}\text { El inglés es útil para conocer otras formas de } \\
\text { vida, etc. }\end{array}$ & & & & & & & & & & & \\
\hline $\begin{array}{l}\text { El inglés es útil en viajes y estancias en otros } \\
\text { países }\end{array}$ & & & & & & + & & & & + & \\
\hline $\begin{array}{l}\text { El inglés es útil para mis estudios poste- } \\
\text { riores }\end{array}$ & + & + & & + & & + & & & + & & \\
\hline
\end{tabular}

+ Correlación positiva estadísticamente significativa ( $r$ de Pearson ${ }^{3}$ )

- Correlación negativa estadísticamente significativa (r de Pearson) 


\section{a) Me gusta aprender inglés}

Según la prueba $r$ de Pearson, aplicada en este apartado, existe una correlación positiva entre la afirmación me gusta aprender inglés y los siguientes objetivos:

- Producir mensajes orales y escritos

- Reflexionar sobre el funcionamiento de la lengua extranjera

- Reflexionar sobre los propios procesos de aprendizaje

- Apreciar y reaccionar ante los usos imaginativos y creativos de la lengua extranjera

- Apreciar los significados sociales y culturales.

Esto indica que cuanto más le gusta al alumno aprender inglés, mejor es su rendimiento en estos objetivos.

\section{b) Aprender inglés es fácil}

También es positiva la correlación entre aprender inglés es fácil y los objetivos que seguidamente se exponen:

- Adquirir la capacidad de comunicarse

- Comprender textos orales y escritos

- Producir mensajes orales y escritos

- Leer de forma comprensiva y autónoma

- Transferir las estrategias de comunicación

- Utilizar estrategias de aprendizaje y recursos didácticos

- Apreciar y reaccionar ante los usos imaginativos y creativos de la lengua extranjera

- Valorar la importancia del conocimiento de lenguas.

Que los estudiantes valoren que aprender inglés es fácil, favorece un alto resultado en la mayoría de los objetivos.

\section{c) Me gustan las actividades de la clase de inglés}

A mayor grado en la afirmación me gustan las actividades de la clase de inglés, mejor es el resultado académico de los objetivos:

- Apreciar y reaccionar ante los usos imaginativos y creativos de la lengua extranjera

- Apreciar los significados sociales y culturales.

\section{d) El inglés es útil útil en viajes y es- tancias en otros países}

El que el alumnado estime que el inglés es útil en viajes y estancias en otros países, incide positivamente en la consecución de los objetivos:

- Reflexionar sobre el funcionamiento de la lengua extranjera

- Apreciar los significados sociales y culturales

- De tal manera, que a mayor grado de estimación, mayor rendimiento en estos objetivos.

\section{e) El inglés es útil para mis estudios posteriores}

La afirmación el inglés es útil para mis estudios posteriores establece una correlación positiva con los objetivos:

- Adquirir la capacidad de comunicarse

- Comprender textos orales y escritos en lengua extranjera

- Leer de forma comprensiva y autónoma

- Reflexionar sobre el funcionamiento de la lengua extranjera

- Apreciar y reaccionar ante los usos imaginativos y creativos de la lengua extranjera.

Una alta estimación de las preguntas planteadas repercute, positivamente, en el logro de dichos objetivos. 


\section{Conclusiones}

Conforme con los resultados anteriormente expuestos, podemos extraer las siguientes conclusiones en relación con la consecución de los objetivos de esta investigación:

Conocer las creencias del alumnado con respecto a la lengua inglesa

A la mayor parte del alumnado $(73,5 \%)$ le gusta algo o bastante aprender inglés, aunque tiene dificultad con su aprendizaje. Un 44,7\% lo considera nada o poco fácil. En cuanto a las actividades de la clase de inglés, las opiniones están divididas, principalmente, entre aquellos a quienes les gustan poco $(31,9 \%)$ y a los que les gusta algo (36,2\%).

En general, la mayoría del alumnado considera que aprender inglés es bastante o muy útil para conocer otras formas de vida, para viajar, para vivir en otros países y para sus estudios posteriores.

Establecer correlaciones entre las creencias del alumnado y su desarrollo académico según los siguientes objetivos curriculares:

Las creencias más influyentes en el rendimiento académico y, por consiguiente, en la consecución de los objetivos curriculares son las valoraciones sobre la facilidad de aprender inglés, la utilidad de la lengua inglesa para los estudios posteriores del alumnado y el gusto por el estudio del inglés. Una creencia firme y alta en estas afirmaciones incide extraordinariamente en un mayor rendimiento académico.

Las creencias sobre el agrado por las actividades realizadas en clase de inglés y que el inglés sea útil en via- jes y estancias en otros países influyen positivamente, pero en menor medida que las expuestas anteriormente, en la consecución de los objetivos curriculares. La única creencia que no mantiene correlación con el logro de objetivos es la valoración que afirma que el inglés es útil para conocer otras formas de vida, etc.

Finalmente, conviene recalcar la gran influencia que ejercen las creencias del estudiante en la consecución de los objetivos. Esto está estrechamente vinculado a la motivación intrínseca del alumnado, por la que el individuo experimenta un deseo de ejecutar una conducta y de ser efectivo por y para su propio interés. Además, esta motivación interna anima al individuo a enfrentarse a nuevos retos y a buscar novedades.

\section{Notas}

1 BALLI (Befiefs About Language Learning Inventory) es una batería de pruebas diseñadas por Oxford en 1990 para medir las creencias de los estudiantes de lenguas extranjeras. Se compone de cincuenta ítems divididos en seis grupos, que atienden a las destrezas de aprendizaje de la memoria, cognitivas, compensatorias, metacognitivas, afectivas y sociales. Utiliza una escala Likert graduada para su medición.

2 Coeficiente de fiabilidad estadística Alfa de Cronbach es una prueba estadística basada en análisis de correlaciones que indica el grado de fiabilidad estadística de las respuestas a una escala de un número determinado de ítems. Sus valores límites están entre 0 y 1 .

3 Coeficiente de correlación $r$ de de Pearson es un coeficiente que mide el 
grado de asociación de dos variables escalares. Vale 0 cuando la asociación es nula y 1 cuando ambas variables son iguales.

\section{Bibliografía}

Azjzen, I. y Fishbein, M. (2000). Attitudes and attitude-behavior relation: reasoned and automatic processes, en Stroebe, W. y Hewstone, M., (Eds.). European Review of Social Psychology. Vol. 11, John Wiley \& Sons, pp. 1-33.

Basturkmen, H., Loewen, S. y Ellis, R. (2004). Teachers' Stated Beliefs about Incidental Focus on Form and Their Classroom Practices. Applied Linguistics, 25, 2, 243-272.

Barcelos, A. M. F. (1995). Cultura de aprender língua estrangeira (inglês) de alunos de Letras. Dissertação (Mestrado em Lingüística aplicada). Universidade Estadual de Campinas, Campinas, 1995. (2000). Understanding Teachers' and Students' Language Learning Beliefs in Experience: A Deweyan Approach. Tesis doctoral en enseñanza del inglés como segunda lengua. Tuscaloosa: College of Education, The University of Alabama. (2006). Cognição de profesores e alunos: Tendências recentes na pesquisa de crenças sobre ensino e aprendizagem de línguas, en Barcelos, A. M. F. y Vieria Abrahão, M. H. (orgs.) Crenças e ensino de línguas: foco no profesor, no aluno e na formação de profesores. Campinas: Pontes Editores, pp. 15-42.

Cohen, L. y Manion, L. (1990): Métodos de Investigación Educativa. Madrid: La Muralla.
Corpas Arellano, M. D. (2008). Estrategias de aprendizaje: la memoria en la adquisición de la lengua inglesa. Contextos Educativos, 11, pp. 2332. Universidad de La Rioja. (2010). La mujer y las estrategias de aprendizaje en la adquisición de la lengua inglesa. Revista Nebrija de Lingüística Aplicada a la Enseñanza de las Lenguas, 8, 4, pp. 3-16.

(2011). Las expectativas y el rendimiento académico en el aprendizaje del inglés. Aula de innovación educativa, 207, pp.57-60.

Diab, R.L. (2000). Lebanese students' beliefs about English and French. A study of university students in a multilingual context. Dissertation Abstracts International, 62, 2, pp. 497-521.

Espinosa Taset, I. (2009). Las creencias de aprendizaje de principiantes brasileños sobre la escritura en la adquisición del E/LE. Revista Nebrija de Lingüística Aplicada a la Enseñanza de las Lenguas, 6, pp. 1- 14.

Holec, H. (1981). Autonomy and Foreign Language Learning. New York: Pergamon Press.

Horwitz, E.K. (1987). Surveying students beliefs about language learning, en Wenden, A. y Rubin, J. (eds.). Learner Strategies in Language Learning. London: PrenticeHall, pp. 119-129.

(1999). Cultural and situational influences on foreign language learners' beliefs about language learning: A review of BALLI studies. System, 27, 557-576.

Kern, G. (1995). Students' and teachers' beliefs about language learning. Foreign Language Annals, 28, 1, pp. 71-92. 
Kunt, N. (1997). Anxiety and beliefs about language learning: A study of Turkish-speaking university students learning English in North Cyprus. Dissertation Abstracts International, 59, 1, pp. 111-137.

Ortega y Gasset, J. (1959). Ideas y Creencias. (6 ${ }^{a}$ ed.). Madrid: EspasaCalpe.

Peacock, M. (1999). Beliefs about language learning and their relationship to proficiency. International Journal of Applied Linguistics, 9, 2, pp. 247-266.

(2001). Pre-service ESL Teachers' beliefs about second language learning: a longitudinal study. System, 29, 2, pp. 177-195.

Ramos Méndez, C. (2010). Las creencias de los alumnos: posibles im- plicaciones para el aula de español como lengua extranjera. Monográficos Marco ELE, 10, pp. 105-114. Richardson, V. (1996). "The role of attitudes and beliefs in learning to teach", en Sikula, J., Buttery, T. J. y Guyton, E. (eds.). Handbook of Research on Teacher Education. $2^{\mathrm{a}}$ ed. New York: Macmillan, pp. 102119.

Williams, M. y Burden, R. (1999). Psicología para Profesores de Idiomas: Enfoque del Constructivismo Social. Cambridge: Cambridge University Press.

Woods, D. (1996). Teacher Cognition in Language Teaching: Beliefs, Decisions-making and Classroom Practice. Cambridge: Cambridge University Press. 
